\title{
Renal adenocarcinoma-associated erythrocytosis in a cat: clinicopathological features and immunohistochemical results
}

\author{
Roberta Troia ${ }^{1,{ }^{*}}$, Chiara Agnoli ${ }^{1}$, Federico Fracassi ${ }^{1}$, Giuliano Bettini ${ }^{1}$, Alessandra Sfacteria ${ }^{2}$, Luciano Pisoni $^{1}$ and \\ Francesco Dondi ${ }^{1}$ \\ ${ }^{I}$ Department of Veterinary Medical Sciences, Alma Mater Studiorum-University of Bologna, Italy \\ ${ }^{2}$ Department of Veterinary Sciences, University of Messina, Italy
}

\begin{abstract}
This report documents a case of secondary inappropriate erythrocytosis in a cat with renal cell adenocarcinoma, its stabilization through manual erythrocytapheresis, and the EPO-immunostaining on the affected kidney. An 11-yearold cat was presented with lethargy, weight loss and polyuria/polydipsia. An abdominal mass was detected upon physical examination. Clinicopathological work-up revealed marked erythrocytosis (HCT value 64.8\%), renal azotemia and decreased urine specific gravity (USG). An abdominal ultrasound was performed, localizing the mass in the right kidney. Serum erythropoietin (EPO) was above the reference interval (RI), and the cytology of the mass was indicative of renal carcinoma. Manual erythrocytapheresis was performed in order to stabilize the patient before surgery, improving the cat's clinical and clinicopathological condition. After nephrectomy, EPO and creatinine concentrations returned within the RI, while the USG markedly increased. Histopathology confirmed the diagnosis of renal adenocarcinoma. Immunohistochemistry with anti-EPO antibody revealed diffuse and strong cytoplasmatic positivity in tumor cells.
\end{abstract}

Keywords: Erythropoietin, Feline, Immunohistochemistry, Manual erythrocytapheresis, Renal neoplasia.

\section{Introduction}

Erythrocytosis is defined as the relative or absolute increase in red blood cells (RBC) count above the reference interval (RI) (Nitsche, 2004). Dehydration or body fluids shift are the main reasons for relative erythrocytosis, which resolves after appropriate fluid therapy. Absolute erythrocytosis can be either primary or secondary. Primary erythrocytosis consists of a myeloproliferative disorder occurring independently of erythropoietin (EPO) production, while secondary erythrocytosis develops as a consequence of increased EPO concentrations (EPO-dependent erythrocytosis). The latter can be further classified into appropriate or inappropriate. Secondary appropriate erythrocytosis arises in response to persistent systemic hypoxia, which is absent in the inappropriate form (Nitsche, 2004). Secondary inappropriate erythrocytosis (SIE) occurs in association with different neoplasms and renal diseases (Kessler, 2008). In particular, a link between renal neoplasia and SIE has been widely reported in humans and dogs (Hodges et al., 2007; Durno et al., 2011; Osumi et al., 2013;), but has only occasionally described in cats (Hasler and Giger, 1996; Henry et al., 1999; Yuki et al., 2005; Klainbart et al., 2008; Noh et al., 2013). Different types of renal tumors including carcinoma, lymphoma and fibrosarcoma have been related with SIE in dogs (Gorse, 1988; Crow et al., 1995; Durno et al., 2011), while an association with renal adenocarcinoma has been reported in feline patients (Yuki et al., 2005; Klainbart et al., 2008; Noh et al., 2013).

This report describes a case of SIE in a cat affected by renal cell adenocarcinoma, the patient stabilization through manual erythrocytapheresis, and the EPOimmunostaining on the affected kidney.

\section{Case Details}

An 11-year-old, $6 \mathrm{Kg}$, neutered male domestic shorthair cat was referred for lethargy, weight loss, occasional vomiting and polyuria/polydipsia lasting for three weeks. The cat had no history of previous medical problems. Upon physical examination, a body condition score of $7 / 9$, depressed mental status and bright pink mucous membranes were noticed. No signs of clinically detactable dehydration were present. An irregular mass was palpated in the cranial right side of the abdomen. Non-invasive blood pressure (BP) measurement using a veterinary oscillometric device (petMAP ${ }^{\mathrm{TM}}$ graphic, Ramsey Medical, Inc., Tampa, F, USA) revealed high-risk hypertension (210/110 mmHg). Complete blood count (CBC) (ADVIA 2120, Siemens Healthcare Diagnostics, Tarrytown NY, USA) revealed marked erythrocytosis with increased RBC $\left(16.2 \times 10^{6} / \mu \mathrm{L}, \mathrm{RI} 5.0-10.0 \times 10^{6} / \mu \mathrm{L}\right)$, hematocrit (HCT) (64.8\%, RI 24-45\%) and hemoglobin concentration (20.6 g/dL, RI 8.0-15.0 g/dL) above the RI. Complete hematologic profile is reported in Table 1. 
Table 1. Hematologic profile of a cat with secondary inappropriate erythrocytosis associated to renal adenocarcinoma.

\begin{tabular}{lcc}
\hline Variable & Patient's Value Reference Interval \\
\hline Hematocrit $(\%)$ & 64.8 & $24-45$ \\
RBC $\left(10^{6} / \mu \mathrm{L}\right)$ & 16.2 & $5.0-10.0$ \\
Hemoglobin & 20.6 & $8-15$ \\
$(\mathrm{~g} / \mathrm{dL})$ & 40 & $39-55$ \\
MCV $(\mathrm{fL})$ & 31.8 & $30-36$ \\
MCHC $(\mathrm{g} / \mathrm{dL})$ & 7440 & $5000-19000$ \\
WBC $(/ \mu \mathrm{L})$ & 5090 & $2000-12500$ \\
Neutrophils $(/ \mu \mathrm{L})$ & 1590 & $1500-7000$ \\
Lymphocytes & & \\
$(/ \mu \mathrm{L})$ & 85000 & $300000-700000$ \\
Platelets $(/ \mu \mathrm{l})$ & 17.9 & $10-15.5$ \\
MPV $(\mathrm{fL})$ & &
\end{tabular}

The evaluation of the blood film revealed mild anisocytosis and minimal polychromasia, numerous platelet clumps and large platelets. Chemistry profile (OLYMPUS AU 400, Beckman Coulter/OLYMPUS, Munich, Germany) showed azotemia (creatinine 2.5 $\mathrm{mg} / \mathrm{dL}$, RI 0.8-1.8; urea $104 \mathrm{mg} / \mathrm{dL}$, RI 15-60) and mild hyperproteinemia (total protein $8.35 \mathrm{~g} / \mathrm{dL}$, RI 6-8; albumin $3.85 \mathrm{~g} / \mathrm{dL}$, RI 2.90-3.60). Ionized calcium results and coagulation profile were within the RI.

A free-catch midstream urine specimen was submitted to urinalysis, which revealed a urinary specific gravity (USG) of 1024, pH of 5.5 and unremarkable sediment. The urinary protein to creatinine ratio was normal $(0.1$; RI 0-0.4). Thoracic radiographs were unremarkable. Abdominal ultrasound showed a $4.6 \times 2.3 \mathrm{~cm}$, round and patchy mass in the cranial pole of the right kidney. An ultrasound-guided fine-needle aspiration (BD Spinal needle 25 GA 3.00 IN 0.50 x 75 mm, Becton Dickinson SA, S. Augustin del Guadalix Madrid, Spain) of the mass was performed, and smears were stained with May Grunwald-Giemsa (Merck KGaA, 64271 Darmstadt, Germany). Cytologic samples were markedly hemodiluted, and displayed threedimensional clusters of moderately pleomorphic epithelial cells with variable nucleus/cytoplasmic ratio, round to oval central nuclei with lacy reticular chromatin and prominent nucleoli (Fig. 1). Although anisocytosis and anisokaryosis were mild, cytology was considered consistent with carcinoma, and presumptive diagnosis was renal cell carcinoma. Differential diagnoses included transitional cell carcinoma of the renal pelvis and metastatic carcinoma. A high performance liquid chromatography method (High performance liquid chromatography, Water Corp HPLC system, Waters PDA Detector [Water Corp., Milford, MA, USA], Acquity UPLC System [Waters Corporation, Manchester England]) for erythropoietin (EPO) measurement in serum sample was used for the case reported.

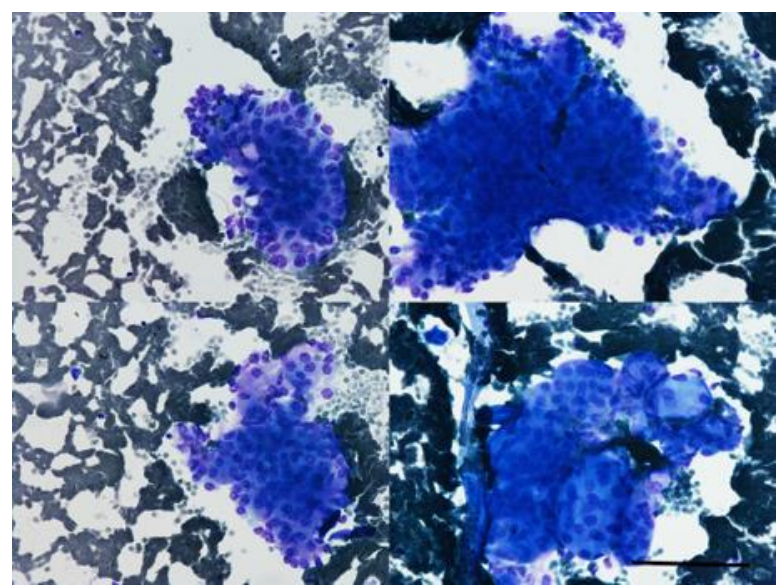

Fig. 1. Fine-needle aspirate from a renal mass in a cat. Poorly cellular and hemodiluted sample, with large three-dimensional clusters of moderately pleomorphic epithelial cells with variable nucleus/cytoplasmic ratio, moderate anisokariosis and prominent nucleoli (MayGrunwald-Giemsa stain; Bar $=50 \mu \mathrm{m}$ ).

The sample was analyzed in liquid chromatography coupled UV detection. EPO measured upon admission in our case was above the RI $(0.54 \mathrm{mUI} / \mathrm{mL}$, RI 0.39 0.51). These findings supported the diagnosis of SIE associated with renal neoplasia, in response to abnormal production of EPO or EPO-like substances. The cat was hospitalized and stabilized with intravenous fluids and supportive care. Since the lack of clinically evident dehydration and the presence of high-risk systemic hypertension, a conservative fluid therapy regimen (Lactate Ringer's solution, $2 \mathrm{~mL} / \mathrm{kg} / \mathrm{h}$ ) was elected. A re-evaluation of the packed cell volume (PCV) after 10 hours of fluid therapy administration showed no significant change $(63 \%)$. Due to the persistence of marked erythrocytosis and the lack of clinical improvement, manual erythrocytapheresis was performed: $15 \mathrm{~mL} / \mathrm{kg}$ of whole blood were drawn from the jugular vein, similarly to previously reported (Lucas et al., 2004). Collected blood (Terumo CPDA-1 Double Blood Bag, Terumo Europe N.V., Interleuvenlaan 40, 3001 Leuven, Belgium) was processed using a refrigerated centrifuge (CRIOFUGE 6000i, Heraeus, Kendro Laboratory Products GmbH, Langenselbold, Germany) (3500g for 10 minutes including acceleration time, temperature: $\left.+4-6^{\circ} \mathrm{C}\right)$. After separation (Separation Stand, Terumo Corporation, Hatagaya, Shibuya-ku, Tokyo, Japan), $30 \mathrm{~mL}$ of fresh plasma were returned to the patient.

A marked improvement in the general condition of the cat was noticed, and BP reduced to $160 / 90 \mathrm{mmHg}$. After this procedure, the PCV was significantly reduced (53\%; RI 24-45\%). The patient was discharged, and re-hospitalized in view of surgery two weeks later. Blood exams at that time revealed a HCT of $56.7 \%$, and a significant reduction in serum 
creatinine concentration compared to the previous baseline $(1.98 \mathrm{mg} / \mathrm{dL}$, RI $0.8-1.8)$. Due to the type of lesion, a nephrectomy of the right kidney was performed. Grossly, the resected kidney revealed a $4 \mathrm{x}$ $2.5 \times 3 \mathrm{~cm}$ reddish mass at the cranial pole, noncapsulated, irregularly lobulated, invading both the cortex and the medulla (Fig. 2).

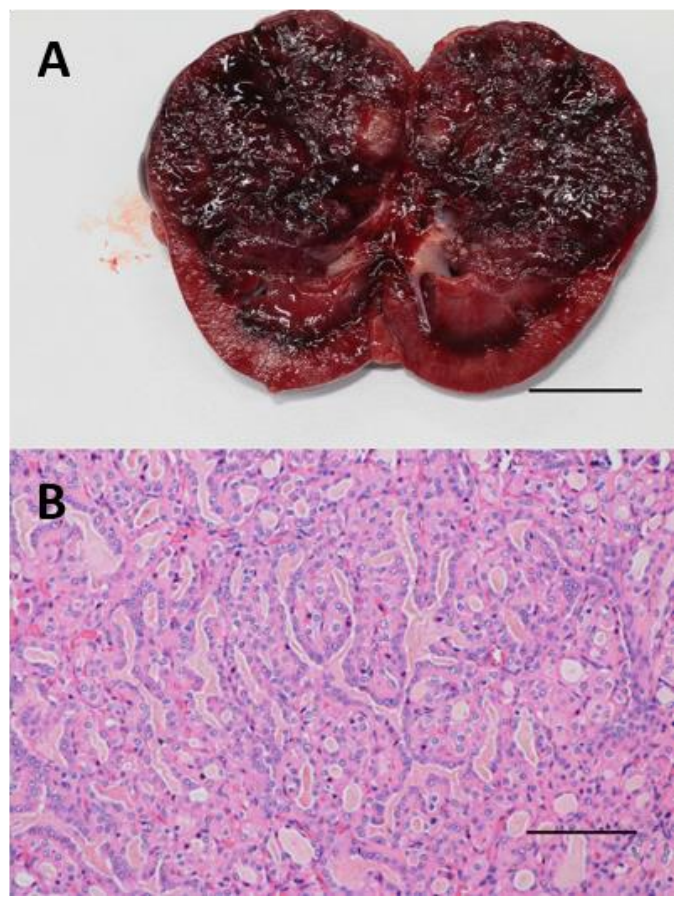

Fig. 2. Gross appearance and histopathology of a feline renal mass. (A): Surgically resected kidney. The tumor mass has completely invaded cortex and medulla of the cranial pole; on section, the tumor tissue is noncapsulated and composed of multiple red lobules embedded in a dark red gelatinous tissue (Bar=2.5 cm). (B): Moderately pleomorphic epithelial cells arranged in irregular tubules and papillae, consistent with tubulopapillary renal cell carcinoma (Hematoxylin and eosin stain; Bar=50 $\mu \mathrm{m})$.

The histologic examination of hematoxylin and eosin stained sections from selected portions of the tumor mass demonstrated tubular and papillary formations of neoplastic epithelial cells, with cuboidal to columnar shape, eosinophilic cytoplasm, vesicular nuclei and prominent nucleoli. The diagnosis was well differentiated tubulo-papillary renal cell carcinoma.

Replicate sections were then immunostained with an anti-EPO antibody (Rabbit IgG polyclonal anti-EPO antibody, clone H-162, Santa Cruz Biotechnology, Inc.) using the labeled avidin-biotin (LAB). In detail, slides were steamed in $0.01 \mathrm{moL} / \mathrm{L}$ sodium citrate buffer, $\mathrm{pH}$ 6, in a microwave oven. Endogenous peroxidase activity was quenched by $0.3 \%$ hydrogen peroxide in methanol while non-specific binding of immunoglobulins and tissue proteins were blocked by incubation with either $5 \%$ non-fat dry milk or $2.5 \%$ goat normal serum. Slides were then incubated overnight at $4{ }^{\circ} \mathrm{C}$ with an anti-EPO antibody at a final concentration of 1:200, followed by incubation at room temperature with a goat anti-rabbit biotinylated $\mathrm{IgG}$ (Goat anti-rabbit biotinylated IgG, Biospa, Milan, Italy) and with an avidin peroxidase complex (Avidin peroxidase complex, Biospa, Milan, Italy). The reaction was developed with the DAB Peroxidase (HRP) Substrate Kit, 3.3'-diaminobenzidine (DAB Peroxidase HRP Substrate Kit, 3.3'-diaminobenzidine, Vector Laboratories Inc. Burlingame, CA, USA) and counterstained with hematoxylin. Negative controls were also performed by omission of primary antibodies, substitution of primary antibodies with normal serum, substitution of primary antibodies with non-reactive antibodies of the same species and immunoglobulin class. Replicates were also developed using a horseradish peroxidase (HRP) secondary antibody to confirm results and exclude nonspecific biotin bound. The immunohistochemical stain was interpreted by assessing the intensity of labeling. Cytoplasmic and/or membrane immunoreactivity was considered positive. In the case here reported, an intense and diffuse cytoplasmic positivity of tumor cells was demonstrated (Fig. 3).

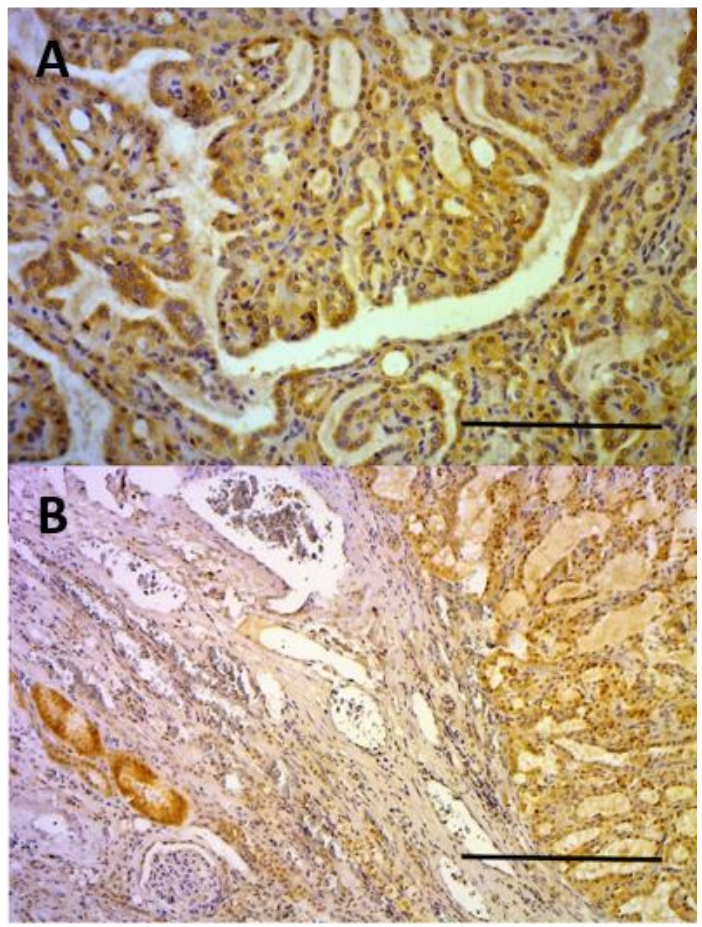

Fig. 3. Immunohistochemistry for erythropoietin (EPO) expression. Diffuse and intense cytoplasmic immunostain for EPO in tumor cells (A: Bar=50 $\mu \mathrm{m})$ and in tubular cells of non-neoplastic renal tissue adjacent to the carcinoma (B: Bar=100 $\mu \mathrm{m})$. 
The cat remained hospitalized in order to receive supportive therapy and clinical and clinicopathological monitoring.

Over the postoperative period (day 2), a gradual decline in PCV and serum creatinine concentration was observed (38\%, RI 24-45\%; 1.92 mgdL, RI 0.8-1-8 $\mathrm{mg} / \mathrm{dL}$, respectively). CBC was within the RI (RBC $9.3 \times 10^{6} / \mu \mathrm{L}$, RI $5.0-10.0 \times 10^{6} / \mu \mathrm{L}$; HCT $35.4 \%$, RI $24-$ 45\%; hemoglobin concentration $11.5 \mathrm{~g} / \mathrm{dL}$, RI 8.015.0), and serum creatinine and EPO concentrations decreased until normalization $(1.76 \mathrm{mg} / \mathrm{dL}$, RI 0.8-1.8 and $0.28 \mathrm{mUI} / \mathrm{mL}$ RI $0.39-0.51$; respectively) in the next two weeks. The cat remained clinically stable for eight months postoperatively; additional re-checks showed the resolution of the polyuria/polydipsia (USG of 1080), and CBC and chemistry profile results were within RI.

At nine months postoperatively, the cat was evaluated for anorexia and mental disorientation. Physical examination revealed anisocoria and multiple cranial nerve defects. BP was $190 / 100 \mathrm{mmHg}$, while the fundus examination showed no abnormalities. Thoracic radiographs, abdominal ultrasound, $\mathrm{CBC}$ and chemistry were unremarkable.

Metastatic intracranial disease was suspected but the owner declined further imaging studies. The patient's conditions progressively worsened until euthanasia was elected. Due to the owner's objection, necropsy was not performed.

\section{Discussion}

In this report, a case of SIE in a cat affected by renal cell adenocarcinoma has been documented, with a main focus on the patient stabilization through manual erythrocytapheresis and the EPO-immunostaining on the neoplastic kidney.

SIE associated with renal neoplasia is an uncommon condition described in cats, perhaps because of the rare occurrence of primary renal tumors in this species (Henry et al., 1999; Klainbart et al., 2008).

EPO measurement in absolute erythrocytosis could be useful in differentiating primary (low to normal EPO concentration) from secondary erythrocytosis (high EPO concentration) (Nitshce, 2004; Hodges et al., 2007).

In the current case, the finding of erythrocytosis with elevated EPO concentration and the evidence of a renal adenocarcinoma indicated SIE associated with the neoplasia. In two cases of renal carcinoma-associated erythrocytosis in cats, EPO measured by ELISA resulted within the RI (Klainbart et al., 2008). In a similar scenario, EPO measured by radio-immunoassay was elevated (Noh et al., 2013). Differences in terms of the technique used, cyclic EPO production or the presence of EPO-like substances that are not measured by the assay used may explain these inconsistent findings.
Other causes of erythrocytosis could be reasonably excluded in our case. The contribution of dehydration on the documented erythrocytosis could not be completely ruled out since the polyuria/polydipsia and the sporadic vomiting reported prior to presentation. However, due to the lack of significant clinical dehydration, the inadequate USG, and the persistence of the abnormalities after fluids administration, a significant component of relative erythrocytosis seems unlikely. An arterial blood gas analysis to exclude the presence of systemic hypoxia as a cause of secondary appropriate erythrocytosis was not performed in the present case for technical reasons. However, there was no clinical or radiographic evidence of cardiopulmonary disease. In addition, normalization of EPO concentration after nephrectomy indirectly demonstrates the link between neoplasia and EPO production.

In human and veterinary medicine, renal tumors are known causes of erythrocytosis, which is considered a paraneoplastic syndrome due to the overproduction of EPO by neoplastic cells or by normal renal parenchyma (peritubular fibroblasts) because of local hypoxia (Nitshce, 2004; Hodges et al., 2007). The latter mechanism is supported by the evidence that nonneoplastic kidney diseases (e.g. cysts, hydronephrosis, and pyelonephritis) or metastatic renal involvement have been associated with SIE in humans and dogs (Hirsch and Leiter, 1983; Kessler, 2008; Pejcic et al., 2011). Primary renal cell carcinoma often overproduces EPO in humans, even if epithelial tubular cells are unable to secrete EPO in normal conditions. However, only a minority of these patients (5\%) develop erythrocytosis; the reason for this remains unclear (Westenfelder and Baranowski, 2000; Hodges et al., 2007; Weisener et al., 2007). Such information in veterinary medicine is lacking.

Immunohistochemistry is one of the techniques used to clarify EPO origin in renal tumors. In the present case, the positive immunohistochemical staining for EPO suggested direct EPO production by tumor cells. Furthermore, immunodetection of hormone expression may be of potential prognostic and therapeutic relevance, since there is some evidence suggesting that EPO signalling through EPO receptors may hasten tumor growth and influences its behaviour stimulating tumor cell replication in vitro and in vivo (Westenfelder and Baranowski, 2000; Nayak-Rao and McCormick, 2013).

Clinical signs related to erythrocytosis are mainly caused by blood hyperviscosity and increased RBC mass, and include hemostatic complications, such as bleeding or thrombotic tendencies, polyuria/polydipsia and neurological abnormalities (Nitsche, 2004; Palagiano and Fracassi, 2013). In our case, the main complaint reported upon admission could have been 
due to erythrocytosis. Azotemia and the inappropriate USG observed on the first chemistry profile were unlikely related to the monolateral neoplasia, but eventually to altered renal blood flow and hyperviscosity. The improvement in renal function (creatinine concentration and USG) after manual erythrocytapheresis and nephrectomy could support this statement. Variable degree of renal impairment or dysfunction with azotemia and different urinary findings (hematuria, proteinuria and reduced urine concentrating ability) have been previously described in cases of SIE associated with renal neoplasia (Gorse, 1988; Crow et al., 1995). These findings highlight the strong impact of erythrocytosis, even if mild or moderate, on kidney perfusion and function, as a consequence of abnormally increased RBC mass and hyperviscosity (Nitsche, 2004). Blood viscosity measurements could have been useful in this case; however, this was not performed.

Therapy for erythrocytosis is different according to the underlying aetiology (Nitsche, 2004; Hodges et al., 2007). Differentiating relative from absolute erythrocytosis is imperative since the extremely different clinical management. Cases of absolute appropriate erythrocytosis in response to systemic hypoxia are unique patients with regard to treatment; in these conditions a moderate degree of erythrocytosis represents a compensatory mechanism to face tissue hypoxia, and phlebotomy is not indicated. Therapeutic phlebotomy is recognized as the emergency treatment for primary erythrocytosis, where the increased RBC mass is considered detrimental, and particularly if clinical signs are present (Nitsche, 2004; Liu et al., 2013).

Phlebotomy could also stabilize patients with SIE before the definitive treatment (e.g. surgical removal of the underlying neoplasia or the renal lesion) is performed (Nitsche, 2004; Kessler, 2008; Klainbart et al., 2008; Liu et al., 2013).

Repeated phlebotomies to treat absolute erythrocytosis may lead to hypovolemia, hypoalbuminemia and coagulation factor deficiency (Nitsche, 2004; Liu et al., 2013). These potential complications should be avoided in critically ill patients, especially before surgery, and the volume losses should be replaced with intravenous fluids or plasma compounds.

In the current case, manual erythrocytapheresis was performed, removing an adequate amount of whole blood and returning the autologous plasma to the patient.

This transfusion technique is feasible in any veterinary centre that routinely provides transfusion treatments; in our case, this procedure was considered safe and useful in view of the surgical treatment of this patient, thus representing a reasonable option when compared to conventional phlebotomy.
Automated extracorporeal apheresis is routinely used in humans with similar disorders (Liu et al., 2013), but has rarely been described in veterinary medicine due to specific requirements, elevated cost and size and volume limitations (Posner et al., 2013). Nevertheless, automated apheresis could also represent a potential promising therapy in veterinary patients in the future. Prognosis for erythrocytosis is strictly related to the underlying disease (Nitsche, 2004). Nephrectomy or tumor excision is the main treatment option for kidney neoplasia, and should rapidly treat renal tumorassociated SIE (Henry et al., 1999; Klainbart et al., 2008; Noh et al., 2013).

In the case here presented, the CBC returned within RI shortly after surgery, and remained stable at the rechecks performed through the following months. Variable time rates for normalization of RBC parameters, ranging from few days to weeks after surgery, have been noticed in veterinary patients (Kessler, 2008; Klainbart et al., 2008; Noh et al., 2013). Several factors including the degree of surgical bleeding, inflammation, timing of phlebotomies, frequency of monitoring and presence of chronic kidney disease may account for the reported variability, making comparisons hard to perform.

Primary renal carcinoma seems to have a high metastatic rate; however, the reported survival time following surgery ranges from eight months up to one year (Henry et al., 1999; Klainbart et al., 2008; Noh et al., 2013). Life quality in the case here presented was excellent for eight months postoperatively, until the occurrence of neurological abnormalities, which were putatively attributed to intracranial metastatic disease. Since autopsy was not performed, other differential diagnoses could not be excluded, which is a limitation of our follow-up evaluation.

This report describes a case of renal adenocarcinomaassociated SIE in a cat. The diagnosis was achieved excluding other causes of erythrocytosis and with a complete clinicopathological and histological examination. Blood EPO concentration was inappropriately elevated, and EPO origin was characterized by immunohistochemistry for the first time in feline medicine. The clinical usefulness of immunostaining for EPO in renal cell adenocarcinoma, and the diagnostic and prognostic relevance of erythrocytosis and EPO measurement should be further characterized. Manual erythrocytapheresis was safe and effective in the pre-surgical management of this patient, and could be advised in similar settings.

\section{Acknowledgments}

The authors thank Dr M. Caldin from the Labratorio d'Analisi Veterinarie San Marco (Padova, Italy) for his support.

\section{Conflict of interest}

The authors declare that there is no conflict of interests. 


\section{References}

Crow, S.E., Allen, D.P., Murphy, C.J. and Culbertson, R. 1995. Concurrent renal adenocarcinoma and polycythemia in a dog. J. Am. Anim. Hosp. Assoc. 31, 29-33.

Durno, A.S., Webb, J.A., Gauthier, M.J. and Bienzle, D. 2011. Polycythaemia and inappropriate erythropoietin concentrations in two dogs with renal T-cell lymphoma. J. Am. Anim. Hosp. Assoc. 47, 122-128.

Gorse, M.J. 1988. Polycythemia associated with renal fibrosarcoma in a dog. J. Am. Vet. Med. Assoc. 15, 793-794.

Hasler, A.H. and Giger, U. 1996. Serum erythropoietin values in polycythaemic cats. J. Am. Anim. Hosp. Assoc. 32, 294-301

Henry, C.J., Turnquist, S.E., Smith, A., Graham, J.C., Thamm, D.H., O'Beiwn, M. and Clifford, C.A. 1999. Primary renal tumours in cats: 19 cases (1992-1998). J. Feline Med. Surg. 1, 165-170.

Hirsch, I. and Leiter, E. 1983. Hydronephrosis and polycythaemia. Urology. 21, 345-350.

Hodges, V.M., Rainey, S., Lappin, T.R. and Maxwell, A.P. 2007. Pathophysiology of anaemia and erythrocytosis. Crit. Rev. Oncol. Hematol. 64, 139158.

Kessler, M. 2008. Secondary polycythaemia associated with high plasma erythropoietin concentration in a dog with a necrotising pyelonephritis. J. Small. Anim. Pract. 49, 363-366.

Klainbart, S., Segev, G., Loeb, E., Melamed, D. and Aroch, I. 2008. Resolution of renal adenocarcinoma-induced secondary inappropriate polycythaemia after nephrectomy in two cats. J. Feline Med. Surg. 10, 264-268.

Liu, H., Liu, H., Shen, J., Sun, C., Guo, C., Lin, H., Yao, J., Ma, R. and Zhang, M. 2013. A clinical analysis of erythrocytapheresis for the treatment of polycythaemia. Transfus. Apher. Sci. 48, 229-233.

Lucas, R.L., Lentiz, K.D. and Hale, A.S. 2004. Collection and preparation of blood products. Clin. Tech. Small. Anim. Prac. 19, 55-62.
Nayak-Rao, S. and McCormick, B. 2013 Erythropoietin use in patients with cancer: to tread with caution? J. Nephrol. 6, 829-835.

Nitsche, E.K. 2004. Erythrocytosis in dogs and cats: diagnosis and management. Compendium 26, 104 118.

Noh, S., Kang, J.H., Kim, G., Chang, D., Ahn, B., Na, K. and Yang M. 2013. Renal adenocarcinoma associated erythrocytosis in a cat. Pak. Vet. J. 33, 125-127.

Osumi, T., Awazu, M., Fujimura, E., Yamazaki, F., Hashiguchi, A. and Shimada, H. 2013. Leukaemia kidney infiltration can cause secondary polycythaemia by activating hypoxia-inducible factor (HIF) pathway. Eur. J. Paediatr. 172, 829832.

Palagiano, P. and Fracassi, F. 2013. Primary erythrocytosis (polycythaemia vera) in a cat. Veterinaria. 4, 31-36.

Pejcic, T., Hadzi-Djokic, J., Markovic, B. and Naumovic, R. 2011. Resolving erythrocytosis and hypertension after open surgical extirpation of giant renal cyst measuring $30 \mathrm{~cm}$ : case report. Ren. Fail. 33, 249-251.

Posner, L.P., Willcox, J.L. and Suter, S.E. 2013. Apheresis in three dogs weighting $<14 \mathrm{Kg}$. Vet. Anaesth. Analg. 40, 403-409.

Weisener, M.S., Munchenhagen, P., Glaser, M., Sobottka, B.A., Knaup, K.X., Jozefowski, K., Jurgensen, J.S., Roigas, J., Warnecke, C., Grone, H.J., Maxwell, P.H., William, C. and Eckardt, K.U. 2007. Erythropoietin gene expression in renal carcinoma is considerably more frequent than paraneoplastic polycythaemia. Int. J. Cancer 121, 2434-2442.

Westenfelder, C. and Baranowski, R.L. 2000. Erythropoietin stimulates proliferation of human renal carcinoma cells. Kidney Int. 58, 647-657.

Yuki, M., Suzuki, K., Sugimoto, N., Higuchi, T. and Suzuki, H. 2005. Two cases of feline renal cell adenocarcinoma with secondary polycythaemia. J. Japan Vet. Med. Assoc. 58, 480-483. 